http://jmscr.igmpublication.org/home/ ISSN (e)-2347-176x ISSN (p) 2455-0450 crossref DOI: https://dx.doi.org/10.18535/jmscr/v7i9.41

\author{
Dournal Of Medical Science And Clinical Research

\title{
Podocytopathy with SLE: A Report of Two Cases
}

Authors

\section{Hana S. Alahmari ${ }^{1,6 *}$, Mansour Y. Somaily ${ }^{2}$, Mohammed M. Alomair ${ }^{3}$, Tariq E. Aljohani ${ }^{4}$, Abdulrahman K. Alabdulsalam ${ }^{5}$, Mohammed A. Omair ${ }^{6}$ \\ ${ }^{1}$ Rheumatology Unit, Department of Medicine, King Khalid University, Abha, Saudi Arabia \\ ${ }^{2}$ Rheumatology Unit, King Khalid Medical City, Abha, Saudi Arabia \\ ${ }^{3}$ Rheumatology Unit, Department of Medicine, Aseer Central Hospital, Abha, Saudi Arabia \\ ${ }^{4}$ Department of Pathology, Pathology and Laboratory Medicine, King Saud University, Riyadh,}

Saudi Arabia

${ }^{5}$ Department of Pathology, Pathology and Laboratory Medicine, King Abdulaziz Medical city, Riyadh, Saudi Arabia

${ }^{6}$ Rheumatology Unit, Department of Medicine, King Saud University, Riyadh, Saudi Arabia

*Corresponding Author

Dr Hana S. Alahmari

Rheumatology Unit, Department of Medicine, College of Medicine, King Khalid University, Abha, Saudi Arabia

\section{Background}

Lupus podocytopathy is a recently recognised new entity of lupus nephritis characterised by diffuse foot process effacement with or without mesangial expansion. Glomerular proliferation and capillary wall immune deposits were inadequate in the histopathological analysis. Clinically, it is manifesting as nephrotic proteinuria in the majority of reported cases. The knowledge about the epidemiology, precipitant aetiology and choices of management are lacking. Here, we describe 2 cases presented with significant proteinuria secondary to foot process effacement with the absence of immunocomplement deposition or glomerular inflammation.

\section{Introduction}

Systemic lupus erythematosus is a systemic disease that can virtually involve any organ or system in the body. Almost $30 \%$ of SLE patients have renal involvement at the time of the diagnosis, and nearly $50 \%$ with renal participation along the disease course. Renal biopsy from patients with SLE who present with nephritic or nephrotic syndrome rarely show changes that are pathogenetically and morphologically unrelated to SLE. Lupus podocytopathy is an example of un-usual renal involvement of SLE. Several reports have described this subclass as a nephrotic syndrome in adult patients with extensive foot process effacement by electron microscope and minimal mesangial lupus nephritis or mesangioproliferative lupus nephritis, sometimes few mesangial deposits of immunoglobulins or complements will be present. The underlying pathogenesis is not well understood yet. Patients typically presented with massive proteinuria, which may be accompanied by acute kidney injury. The prompt response in most of the 
cases supports the possible minimal change-like damage. Nevertheless, there is an unmet need in all the aspect of the disease ${ }^{(1)}$.

\section{Case Report 1}

A 45-year-old female patient diagnosed to have SLE 14 years back in the setting of malar rash, alopecia, arthritis and positive serology (ANA, Ds DNA). She was doing fine on hydroxychloroquine (HCQ) till a years ago when she presented to the clinic with gradual onset lower limbs swelling. Laboratory results showed a significant proteinuria $2781 \mathrm{mg} / 24$ hour, urine analysis showed RBC 1-2 cells/microliter, WBC 2-3 cells/microliter, standard renal profile and normal $\mathrm{C} 3-\mathrm{C} 4$ counts. Kidney biopsy was taken and described a picture of minimal change glomerulonephritis with mild mesangial expansion. Also, the study was negative for the presence of Immunocomplement deposition by immunofluorescence stain with the absence of capillary basement membrane and tubulointerstitial inflammation. The electron microscope was not available. The patient was treated with Mycophenolate Mofetil 2 grams twice per day and $1 \mathrm{mg} / \mathrm{kg} /$ day oral steroid. Three months later, in urine protein was noticeably reduced in urine to 731 $\mathrm{mg} / \mathrm{dl} /$ day but the patients had a side effect of MFM in the form of significant weight loss, gastritis, and diarrhoea. Azathioprine had been instituted alternatively with low dose prednisolone $10 \mathrm{mg}$. Proteinuria level had peaked again after four months of starting azathioprine to reach $5660 \mathrm{mg} / \mathrm{dl}$. Kidney biopsy performed again, which showed minimal light microscopic changes, the ultrastructural study showed extensive podocyte injury and foot process effacement up to $70 \%$ by the electron microscope (Figure1). The mild mesangial proliferation and focal tubulointerstitial chronic changes. There was no evidence of endocapillary proliferation, subendothelial deposits or glomerular scarring. Immunofluorescence was negative for immunocompetent deposition. Mycophenolic Acid $360 \mathrm{mg}$ twice a day has been instituted again, but unfortunately, proteinuria inadequately responded and remained between 3-4 grams the dose raised to $720 \mathrm{mg}$ twice a day and reevaluation done.

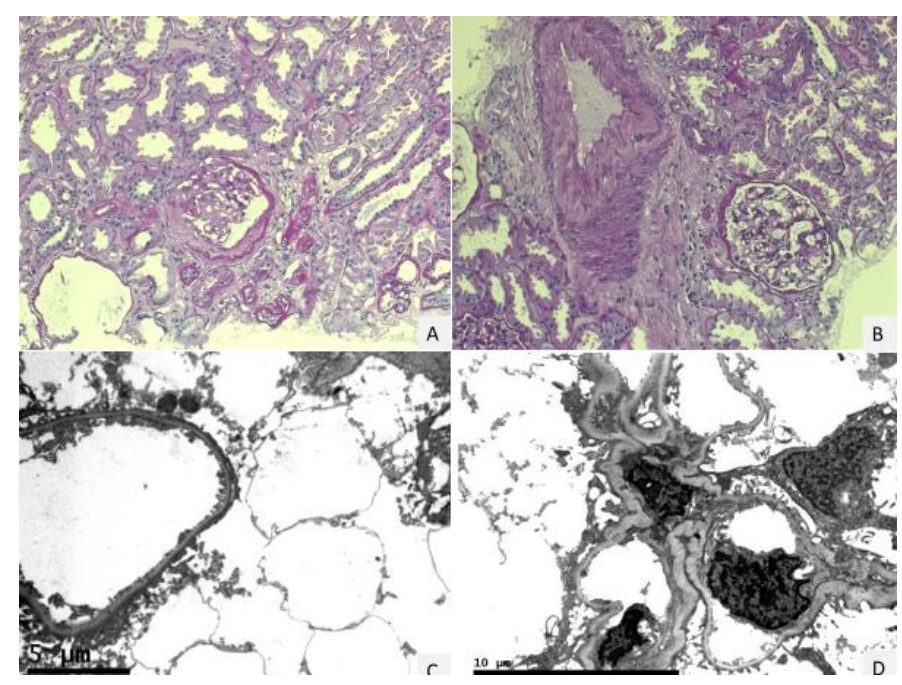

Fig. 1 Kidney biopsy for case report 1 .

\section{Case Report 2}

A 17 years old girl presented to the emergency department with altered sensorium and fever. Before this event, she had several episodes of chondritis and rash over her face and back as well as recurrent mouth and genital ulcers. She has no ear pain, discharges or headache, and she denied a history of upper respiratory tract infection. By examination, she was afebrile, disoriented to time and place. Another neurological examination was unremarkable. Malar rash was observed along with diffuse maculopapular rash over her back. There was no active synovitis or oro-genital ulcers. Laboratory tests showed hemolytic anaemia. Other chemistry panels were normal. CSF examination was within normal values. MRI results were completely normal and cerebral venous vessels were patent. Serology markers revealed high ANA level 1:1280 (speckled pattern), positive dsDNA, low complements and positive lupus anticoagulant. Based on the clinical and laboratory assessment, she was managed as systemic lupus and treated with methylprednisolone $20 \mathrm{mg} 3$ times followed by oral prednisolone $60 \mathrm{mg}$ per day with gradual tapering along with hydroxychloroquine $400 \mathrm{mg}$ a day. Proteinuria was 1.26 grams over 24hour urine collection. Kidney Ultrasound reported normal kidneys size with normal corticomedullary 
differentiation. The patient underwent kidney biopsy that showed mild mesangial expansion and hypercellularity (Figure 2). Immunofluorescence stained for $\operatorname{IgA}$, IgM, IgG, C3, C1q, Kappa and lambda. Capillary loops are patent. There was an increase in mesangial matrix and mesangial electron-dense deposits with few subendothelial electron-dense deposits. Electron microscope examination revealed widespread foot process effacement of epithelial cells. Tubuloreticular inclusions is also noticed. The histopathological analysis described class ii lupus nephritis with extensive foot process effacement. Azathioprine 150 $\mathrm{mg} /$ day was added and discharged home. Follow up visit was 2 months later, and the proteinuria had declined to $320 \mathrm{mg} / 24 \mathrm{hr}$ Patients remained in mild range of proteinuria over 18 months of follow up with normal renal profile and urine analysis.

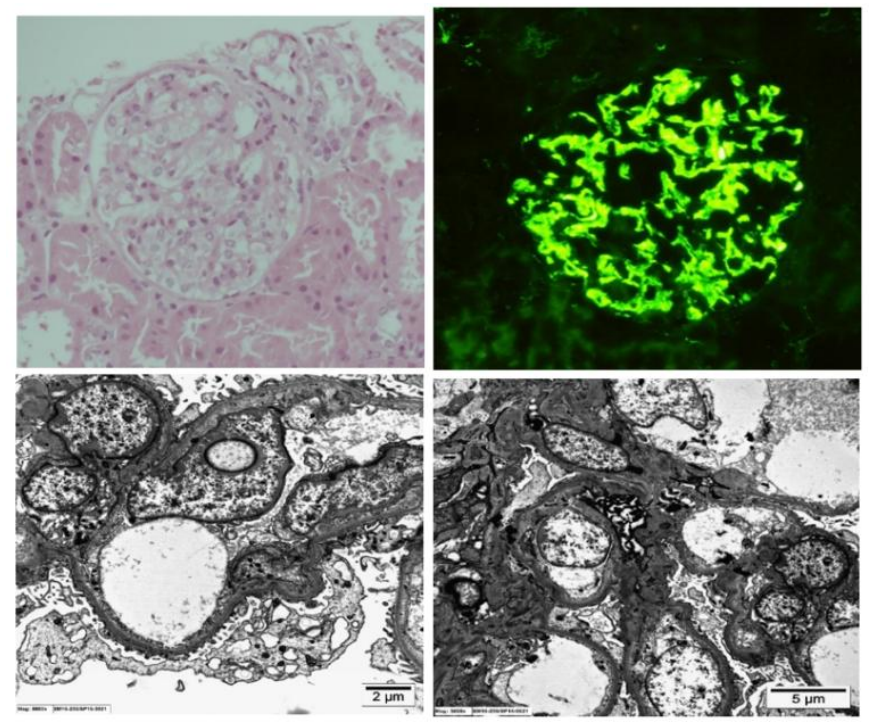

Figure 2: Kidney biopsy for case report 2

\section{Discussion}

Lupus podocytopathy (LP) is clinically presenting with nephrotic syndrome demonstrating minimal change disease (MCD), mesangial proliferation (MsP) or focal segmental glomerulosclerosis (FSGS), while on electronic microscopy, diffuse podocyte foot process effacement with or without sub-epithelial or sub-endothelial deposition. Hu et al. proposed a definition of lupus podocytopathy as follow ${ }^{(2)}$.

1) Clinical: the diagnosis of SLE; nephrotic range proteinuria, often accompanied by acute kidney injury (AKI), and no history of nephrotoxic medications (e.g., NSAIDs) before the onset of renal disease.

2) Light microscopy: minimal glomerular change, mesangial proliferation, or focal segmental glomerulosclerosis patterns without subepithelial or subendothelial deposits. Excluding glomerular scar of proliferative lupus nephritis in the FSGS pattern.

3) Immunofluorescence: the absence of capillary immune deposition with or without Ig and complement deposition in mesangial areas.

4) Electronic microscopy: foot process effacement (FPE) 50\%, typically FPE $>70 \%$ with dense mesangial deposits and in the absence of subepithelial or subendothelial dense deposits, but occasional isolated subepithelial or subendothelial dense deposits may be visible

Researchers from Columbia University College, New York $^{(3)}$ proposed diagnostic criteria for lupus podocytopathy that required no glomerular immunocomplement deposition and $\geq 70 \%$ FPE. If mesangial immunocomplement deposition were observed, the case would be diagnosed as LN-class I with podocytopathy or LN-class II with podocytopathy. However, this stratification has no impaction on the prognosis or treatment options ${ }^{(2)}$. In previous reports, lupus podocytopathy accounted for $0.6-1.5 \%$ of all $\mathrm{LN}$ cases that underwent renal biopsy $^{(4)}$. No differences were found in the age of onset or the male-female ratio compared to other types of LN. The incidence of acute kidney injury in the small number of patients reported was higher than $50 \%{ }^{(5-7)}$ while the incidence was $34 \%$ in the largest cohort, mainly in the FSGN ${ }^{(4)}$. SF Wang et al. studied, 125 biopsies of lupus nephritis and divided into podocytopathy group (defined as podocyte foot process effacement (FPE) $>50 \%$ with nephrotic syndrome (NS)) and mesangial group (FPE $<50 \%$, or FPE $>50 \%$ without NS). They found that the degree of acute tubularinterstitial lesions and renal relapse were significantly higher in the 
podocytopathy group than in the mesangial group ${ }^{(8)}$. The most common hypothesis of underlying pathogenesis suggests that a product of aberrant $\mathrm{T}$ cell function may be responsible for glomerular epithelial cell injury resulting in diffuse podocyte effacement $^{(9)}$. Some authors favour a circulating glomerular toxin hypothesis, where IL-13 overexpression has been suggested to be the main culprit in an experimental study by increasing B7-1, which is produced in a high level in $\mathrm{MCGN}^{(10)}$. Another possible pathology is that Anti-dsDNA antibodies may directly cross-react with podocyte proteins like $\alpha$-actinin- 4 and cause injury ${ }^{(11)}$. The majority of patients with lupus podocytopathy are sensitive to glucocorticoid therapy alone or combined with other immunosuppressive therapies, which is used to treat LN. There is no established evidence regarding the appropriate management and duration of steroid. $\mathrm{Hu}$ et al., found that nearly $90 \%$ of the patients relapsed with glucocorticoid monotherapy, while combination therapy showed a markedly reduced relapse rate by more than $50 \%$ after remission was achieved by glucocorticoid monotherapy or glucocorticoid plus additional immunosuppressive agents ${ }^{(12)}$. Moreover, it had been reported that FSGs was less sensitive to GC therapy with a high relapse rate $^{(7,12)}$. Calcineurin inhibitor agents have been documented to be useful in refractory or steroid-dependent minimal change glomerulonephritis, as well as focal segmental glomerulosclerosis ${ }^{(13)}$. Abatacept also proposed to protect podocytes by blocking the transmembrane protein B7-1 associated with FP effacement ${ }^{(14)}$. However, abatacept has been studied in lupus nephritis, but primary endpoints in randomised clinical trials have not yet been achieved.

\section{Conclusion}

Lupus podocytopathy is a recently recognized new entity of lupus nephritis characterized by diffuse foot process effacement without capillary wall immune deposits and glomerular proliferation. However, the lack of efficient knowledge about the frequency, clinical features and treatment necessitate further investigation.

\section{Conflict of interest: Nil}

\section{References}

1. Fogo AB, Kashgarian M. Diagnostic Atlas of Renal Pathology. 2016.

2. Chen $\mathrm{D}, \mathrm{Hu}$ W. Lupus podocytopathy: a distinct entity of lupus nephritis. J Nephrol. 2018;31(5):629-34.

3. Bomback AS, Markowitz GS. Lupus Podocytopathy: A Distinct Entity. Clin J Am Soc Nephrol. 2016;11(4):547-8.

4. Hu W, Chen Y, Wang S, Chen H, Liu Z, Zeng $\mathrm{C}$, et al. Clinical-Morphological Features and Outcomes of Lupus Podocytopathy. Clin J Am SocNephrol. 2016;11(4):585-92.

5. Huong DL, Papo T, Beaufils H, Wechsler B, Bletry O, Baumelou A, et al. Renal involvement in systemic lupus erythematosus. A study of 180 patients from a single center. Medicine. 1999;78(3):14866.

6. Dube GK, Markowitz GS, Radhakrishnan J, Appel GB, D'Agati VD. Minimal change disease in systemic lupus erythematosus. Clinical nephrology. 2002;57(2):120-6.

7. Kraft SW, Schwartz MM, Korbet SM, Lewis EJ. Glomerular podocytopathy in patients with systemic lupus erythematosus. J Am SocNephrol. 2005;16(1):175-9.

8. SF Wang YC, DQ Chen, ZZ Liu, F Xu, CH Zeng and WX Hu. Mesangial proliferative lupus nephritis with podocytopathy: a special entity of lupus nephritis. 2017.

9. Cunard R, Kelly CJ. T Cells and Minimal Change Disease. Journal of the American Society of Nephrology. 2002;13(5):1409.

10. Lai KW, Wei CL, Tan LK, Tan PH, Chiang $\mathrm{GS}$, Lee $\mathrm{CG}$, et al. Overexpression of interleukin-13 induces minimal-change-like nephropathy in rats. J Am SocNephrol. 2007;18(5):1476-85.

11. Lee SJ, Borsting E, Declèves A-E, Singh P, Cunard R. Podocytes express IL-6 and lipocalin 2/ neutrophil gelatinase-associated 
lipocalin in lipopolysaccharide-induced acute glomerular injury. Nephron Experimental nephrology. 2013;121(34):e86-e96.

12. WX Hu YC, H Bao, ZZ Liu, SF Wang, HT Zhang and $\mathrm{ZH}$ Liu. Glucocorticoid with or without additional immunosuppressant therapy for patients with lupus podocytopathy: a retrospective single-center study. 2015.

13. Meyrier A. Focal and segmental glomerulosclerosis: multiple pathways are involved. Seminars in nephrology. 2011;31(4):326-32.

14. Yu C-C, Fornoni A, Weins A, Hakroush S, Maiguel D, Sageshima J, et al. Abatacept in B7-1-positive proteinuric kidney disease. The New England journal of medicine. 2013;369(25):2416-23. 